\title{
Reliability-Base Performance Measure for Stochastic Networks
}

\author{
Wheyming Tina SONG ${ }^{1}$ \\ Department of Industrial Engineering and Engineering Management, \\ National Tsing Hua University, Hsinchu, 30013, Taiwan
}

\begin{abstract}
An important performance measure for stochastic manufacturing networks is the system reliability, defined as the probability that the production output meets or exceeds a specified demand. This paper reviews and highlights the drawbacks of the previous works, including twenty one archival publications and the most recent published analytical Song rule. The major error in the twenty one archival publications error assumes that entities in the network are discrete and indivisible, while the analysis is based upon continuous flow through the network. That is, they assume that the number of defective items is a real number, instead of a random variable following a binomial distribution. We claim that the analytical approach in Song rule is valid for non-rework case, but is incorrect for general system reliability with reworking when the rework and non-rework path share the same capacity. This note corrects the mistakes in Song rule when the rework and non-rework path share the same capacity. The proposed analytical approach can be easily generalized to general system reliability with multiple reworks. The Song rule and the proposed corrected version of Song rule in this note are computationally inefficient and are only recommended as a validator instead of a problem solver. For big-scale system reliability, the simulation approach are recommended as the problem solver.
\end{abstract}

Keywords. System Reliability, Analytical, Simulation, Binomial Distribution

\section{Introduction}

Consider a stochastic network consisting of a series of $n$ workstations, where the $i$ th workstation $\left(\mathrm{WS}_{i}\right)$ has some random capacity, say $X_{i}$, following a discrete probability distribution. Moreover, the $i$ th workstation produces non-defective items with the nondefective probability $p_{i}$. The value $\theta=P(O \geq d)$, the probability that the random output $O$ of a network meets or exceeds a specified demand $d$, is an important performance measure of stochastic networks such as manufacturing and production systems.

Previous works include 21 archival publications, including recent work ([1]-[3], and [5]) and earlier work referenced in Song rule [8]. All examples adopted in the above-mentioned publications assume the entities to be discrete and indivisible, while the analysis was based upon continuous flow, much like a fluid, through the network. This can result in erroneous conclusions about the system reliability. The most recent work, named the Song rule [8] provided a correct analytical result $\theta$, for non-rework

\footnotetext{
${ }^{1}$ Corresponding Author, Email: wheyming@ie.nthu.edu.tw.
} 
case but is not valid for general system reliability with reworking, especially when the rework path and regular path share the same capacity.

The remainder of this paper is organized as follows. Subsection 1.1 lists all acronyms and notation used in this paper. Subsection 1.2 states the problem definition. Section 2 is a literature review with expansions. Section 3 proposes analytical result for $\theta$ for one rework. The summary and conclusion are given in Section 4.

\section{Background}

\subsection{Acronyms and Notation}

All acronyms and notation, used in this paper, are listed in this Subsection 1.1.1 and 1.1 .2 , respectively.

\subsubsection{Acronyms}

- DES: discrete-events simulation

- $\mathrm{WS}_{i}$ : the $i$ th workstation

- regular path (non-rework path): a network path without rework.

- rework path: a network path relates to rework.

- $\operatorname{rework}(k, r)$ : the rework path where the defective items generated from $\mathrm{WS}_{r}$ are reworked in $\mathrm{WS}_{k}$.

- $\quad$ R.V.: random variable.

- $\operatorname{Ber}(p)$ : A Bernoulli R.V. with parameter $p$.

- cdf: cumulative distribution function.

- C.I.: confidence interval.

- $\quad$ iid: independent identically distributed.

- Song rule: Song captures 4 key words; Stochastic "Output $\geq$ demand" Networks and their Generation.

- $\operatorname{SNLR}(h, w)$ : Stochastic Networks with numbers of productio Lines $h$ in which each line has identical $w$ numbers of Reworks. There is no common WS.

- $\operatorname{SNLR}(1,0)$ : Stochastic Networks without Rework.

- $\operatorname{SNLR}(1,1)$ : Stochastic Networks with one regular path and one rework.

- $\quad$ SNLR-C $(h, w)$ : SNLR with one common WS, and with $h$ lines and identical $w$ reworks in each line; $h=1,2, \ldots ; w=0,1,2, \ldots$.

\subsubsection{Notation}

Without exception, random variables and real values are written as capital and lower case English letter, respectively.

- $\quad$ Pre-determined values (given):

- $\quad n$ : total number of workstations.

- $\quad d$ : demand. 
- $\quad b$ : input value for one path in the stochastic network.

- $\quad B_{0}, b_{0}$ : set $B_{0}=b_{0}=b$.

- $\quad B_{j, 0}$ : input value for the $i$ th path in the stochastic network for $j=1,2$.

- $\quad X_{i}$ : capacity of $i$ th workstation $\mathrm{X}_{i}$ and $\mathrm{X}_{k}$ are independ for any $i \neq k . \mathrm{X}_{i}$ follows a discrete cdf $F x_{i}($.$) .$

- $\quad p_{i}$ : the probability of $\mathrm{WS}_{i}$ 's output is non-defective. Throughout this paper, we name $p_{i}$ the non-defective probability. Note that $p_{i}$ and $\mathrm{X}_{i}$ are irreverent.

- $\quad m$ : total numbers of simulation replications.

- Random Variables that need to be obtained:

- $\quad O$ : output for a network.

- $\quad O_{j}$ : output of the $j$ th line for $j=1,2$.

- $\quad O^{\sharp}$ : output for a regular path of a network with reworking feature. If a network with no rework, $O=O^{\#}$.

- $\quad B_{n}$ : set $B_{n}=O$ for the convenience of analysis.

- $A_{i}$ : input of $\mathrm{WS}_{i}$ for a regular path $\mathrm{A}_{i}=\min \left(B_{i-1}, X_{i}\right)$.

- $\quad B_{i}$ : output from $\mathrm{WS}_{i}$ for a regular path.

- $\quad B_{i} \mid\left(A_{i}=a_{i}\right) \sim \operatorname{binomial}\left(a_{i}, p_{i}\right)$, where $B_{i} \mid\left(A_{i}=a_{i}\right)$ denotes for $B_{i}$ given that input $A_{i}=a_{i}$.

- $O^{*}:$ output for a rework path

- $\quad B_{n}^{*}$ : set $B_{n}^{*}=O^{*}$

- $A_{i}^{*}$ : the input of $\mathrm{WS}_{i}$ for a rework path. $A_{i}^{*}=\min \left(B_{i-1}^{*}, X_{i}^{*}\right)$.

- $\quad B_{i}^{*}: \mathrm{WS}_{i}$ 's output for a rework path. $B_{i} \sim \operatorname{Binomial}\left(a_{i}^{*}, p_{i}\right)$.

- $B_{k-1}^{*}=a_{r}-b_{r}$ : the number of rework items produced from $\mathrm{WS}_{r}$.

- $\quad B_{i}^{*} \mid\left(A_{i}^{*}=a_{i}^{*}\right) \sim \operatorname{binomial}\left(a_{i}^{*}, p_{i}\right)$, where $B_{i}^{*} \mid\left(A_{i}^{*}=a_{i}^{*}\right)$ denotes for $B_{i}^{*}$ given that input $A_{i}^{*}=a_{i}^{*}$.

- $\quad B_{r}^{*}$ : WS ${ }_{r}$ 's output. (Note that $B_{r}^{*} \neq B_{k-1}^{*}=a_{r}-b_{r}$ )

- $X_{i}^{*}=X_{i}-a_{i}$ : the remaining capacity for $\mathrm{WS}_{i}$.

- Real values that need to be obtained:

- $\quad \theta=\mathrm{P}(O \geq d)$ : named "system reliability", the performance measure of the system.

- $\quad o, o^{\#}, o^{*}, a_{i}^{*}, b_{n}, b_{k-1}^{*}, b_{r}^{*}$, and $\hat{\theta}$ : the corresponding real values of , $O$, $O^{\#}, O^{*}, B_{i}, A_{i}^{*}, B_{n}, B_{k-1}^{*}, B_{r}^{*}$, and $\widehat{\Theta}$, respectively.

\subsection{Problem Definition}

Previous work has defined "system reliability $\theta$ " as the probability that the random output $O$ meets the pre-determined constant demand $d$. The goal of this paper is to solve for $\theta$ given the input value is $b$, non-defective probability $p_{i}$ and ramdom capacity $X_{i}$ for networks shown in Figures 1 and 2:

$$
\theta=\mathrm{P}(O \geq d)
$$

where networks illustrated in Figures 1 and 2 are clearly defined below.

The network, shown in Figure 1, is a simple network with one regular path without rework. Each circle in Figure 1 denotes a workstation and the nome of $\mathrm{WS}_{i}$ is listed below the circle for $i=1,2, \ldots, n$. The random capacity $\mathrm{X}_{i}$ and the non-defective 
probability $p_{i}$ of $\mathrm{WS}_{i}$ are listed inside and above the circle, respectively. Throughout this paper, we assume that the random capacity $X_{i}$ and $X_{j}$ are independent, for any $i \neq \mathrm{j}$.

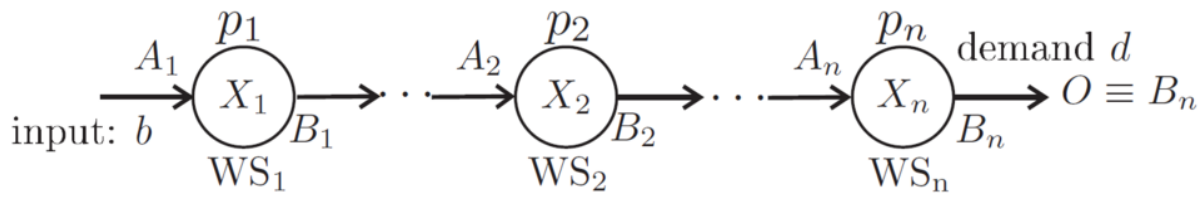

Figure 1. System 1 with one regular path.

The network, shown in Figure 2, is a generalized net work with two paths(one regular and one rework paths), in which (i) is a simple form and (ii) is a decomposition form. The decomposition form, shown in Figure 2 (ii), includes two paths: (a)regular path and (b) rework path. In Figure 2(ii)(b), double circles are used to highlight $\mathrm{WS}_{k}$, and a "square inside a circle" is used to highlight WS $r$ for the rework $(k, r)$, defined in Subsection 1.1.1. The remaining capacity for $\mathrm{WS}_{i}$ is denoted as $X_{i}^{*}, i=k, k+1, \ldots, n$. Again, the parameters $A_{i}$, and $B_{i}, i=1,2, \ldots, \mathrm{n}$ are also listed; and all $A_{i}$ and $B_{i}$, are dependent random variables in that $A_{1} \geq B_{1} \geq \cdots \geq A_{n} \geq B_{n}$. The $A_{j}^{*}$ and $B_{j}^{*}$ are used to illustrate the input and output items for the rework path for $\mathrm{WS}_{j}, j=k$, $k+1, \ldots, n$. Also, $A_{j}^{*}$ and $B_{j}^{*}$ are dependent random variables in that $B_{k-1}^{*} \geq A_{k}^{*} \geq B_{k}^{*} \geq A_{k+1}^{*} \geq$ $B_{k+1}^{*} \ldots \geq A_{n}^{*} \geq B_{n}^{*}$. Particularly, $A_{\mathrm{i}}^{*}=\min \left(B_{i-1}^{*}, X_{i}^{*}\right.$ and $B_{i}^{*} \sim \operatorname{binomial}\left(a_{i}^{*}, p_{i}\right)$. Recall that $B_{k-1}^{*}=a_{r}-b_{r}$ is denoted as the rework items generated from $\mathrm{WS}_{r}$ and will be reprocessed in $\mathrm{WS}_{i}, i=k, k+1, \ldots, n$ according to the associated capacities.

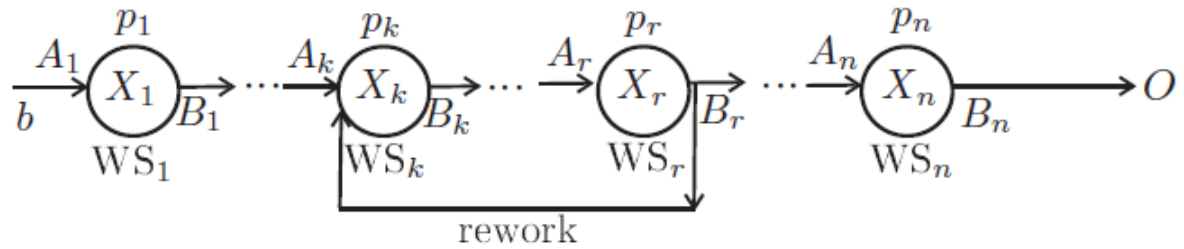

(i) Simple Form

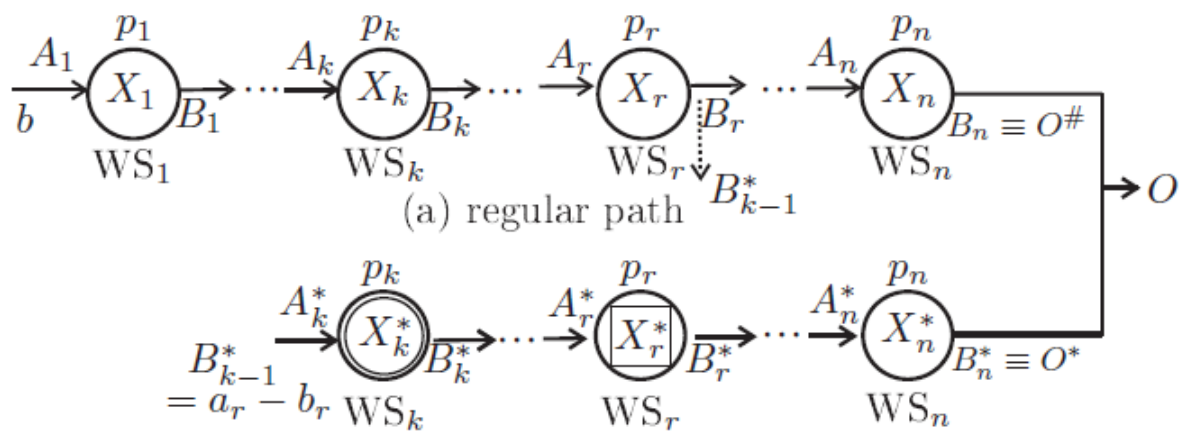

(b) $\operatorname{rework}(k, r)$

\section{(ii) Decomposition Form}

Figure 2. System 2 with (i) simple form and (ii) decomposition form. 
Further explanation of the generalized network rule is given below.

(1) The regular path is the same as the one shown in Figure 1.

(2) The rework items are generated from $\mathrm{WS}_{r}$ and re-processed in $\mathrm{WS}_{i}, i=k$, $k+1, \ldots, n$. That is , the $\operatorname{rework}(k, r)$ starts from $\mathrm{WS}_{k}$ then $\mathrm{WS}_{k+1}$, until the last workstation $\mathrm{WS}_{n}$.

(3) We consider two rules about the the number of rework items re-processed in $\mathrm{WS}_{i}$

- $\quad$ (3.1) depends on the remaining capacity in $\mathrm{WS}_{i}$ after the regular path is completed, where $i=k, k+1, \ldots, n$. Rule (3.1) are adopted by the abovementioned publications, including the 21 archival publications and Song rule.(Note that the Song rule is incorrect under Rule 3.1).

- (3.2) The capacity of regular path and rework path are independent.

(Note that the Song rule is correct under Rule 3.2).

(4) Whether the rework items in $\mathrm{WS}_{i}$ are good or defective depends on the nondefective probability $p_{i}, i=k, k+1, \ldots, n$.

(5) The total output from such generalized network is the sum of the output form regular path and rework $(k, r)$; i.e., $O=O^{\#}+O^{*}$.

(6) The rework process only occurs once. That is, any rework item which is still considered to be defective is discarded after the rework.

\section{Literature Review and Elaboration}

This section reviews 21 archival publications and Song rule [8]. Papers [8] and [9] establishes that twenty-one archival publications, containing twenty three examples, provide incorrect values of the system reliability. The major error assumes that the numbers of defective items is a real number, instead of a random variable following a binomial distribution.

\subsection{Six Network Types in Previous 21 Archival Publications}

To get a global view of various stochastic networks that have been investigated in many archival publications, we summary 6 types of networks in Figure 3: (a) SNLR(1, 1), (b) $\operatorname{SNLR}(1,2)$, (c) $\operatorname{SNLR}(2,1)$, (d) $\operatorname{SNLR}(2,2)$, (e) $\operatorname{SNLR-C}(2,1)$, (f) SNLR$\mathrm{C}(2,2)$; where SNLR and SNLR-C are defined in Subsection 1.1.1. Recall that the parameters inside the parenthesis of SNLR and SNLR-C are numbers of production lines and numbers of reworks. The first 4 examples shown in Figures 3 (a), (b), (c), and (d) are explained clearly in the previous subsections. The last two examples, shown in Figures 3 (e) and (f), are used here to identify the network types studied in the literature, but will not be further investigated in this paper. 


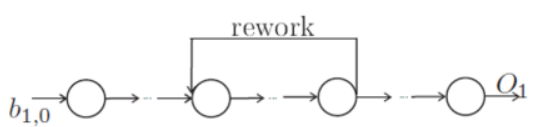

(a) $\operatorname{SNLR}(1,1)$

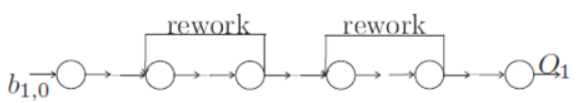

(b) $\operatorname{SNLR}(1,2)$
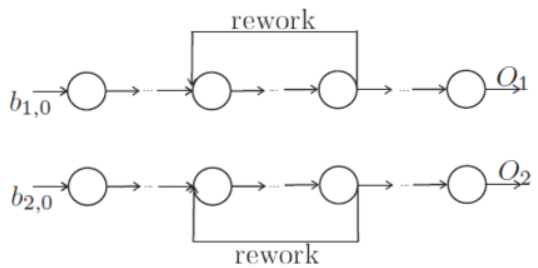

(c) $\operatorname{SNLR}(2,1)$

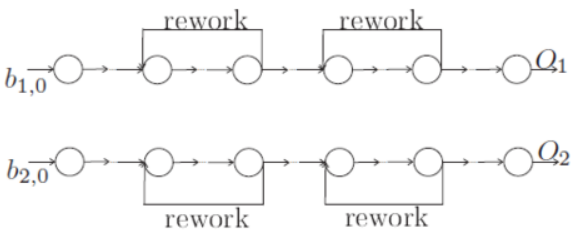

(d) $\operatorname{SNLR}(2,2)$

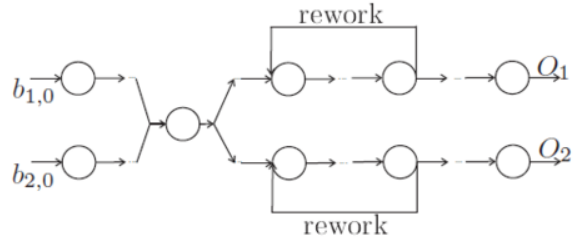

(e) SNLR-C $(2,1)$

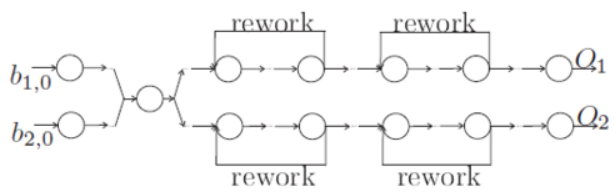

(f) $\operatorname{SNLR-C}(2,2)$

Figure 3. A Summary of 6 types of Stochastic Networks in the Literature.

It is particularly noteworthy that the archival work presents twenty three examples, wherein entities are discrete and indivisible, while the analysis is based upon continuous flow, much like a fluid, through the network. This can result in erroneous conclusions about the system reliability.

Song [8] proved that the $\theta=\prod_{i=1}^{n} \mathrm{P}\left(X_{i} \geq x_{i}\right)$ provided incorrect result for $\theta$. Specifically, the common error is due to the misunderstanding $B_{i} \mid\left(A_{i}=a_{i}\right)$, the no. of good items produced in $\mathrm{WS}_{i}$, given that the corresponding input is $A_{i}=a_{i}, i=1,2, \ldots, n$. This result leads to the conclusion that the previous 21 publications' calculated values are incorrect.

Consider a network of producing PC boards, which are discrete and indivisible. Suppose $\mathrm{WS}_{i}$ 's input is $a_{i}=1$ and the associated non-defective probability is $p_{i}=$ 0.95 . See Figure 3 to distinguish incorrect and correct assumptions about $B_{i}$. Although discrete entities are used in their examples, their analysis is designed for continuous flow, much like a fluid.

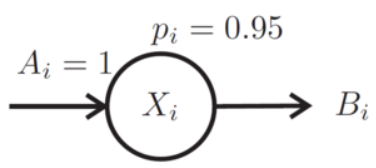

correct: $B_{i}= \begin{cases}1 \text { (non-defective item, }) & \text { with pro. } 0.95 \\ 0(\text { defective item, }) & \text { with pro. } 0.05\end{cases}$

incorrect: $B_{i}=a_{i} p_{i}=0.95$ (unclear meaning)

Figure 4. Correct and Incorrect Versions of $B_{i}$. 


\subsection{Literature II: The Song Rule}

Song rule proposed in [8] and [9] provide an analytical approach for solving $\theta$. The "Song" rule denotes for Simple "Output $\geq$ demand Networks" and its Generations. We claim that Song rule provides correct analytical results for $\operatorname{SNLR}(1,0)$ in general, but not for $\operatorname{SNLR}(1,1)$ in general. Song rule provides correct analytical results for networks with reworking only when the outputs from regular and from rework pathes are independent. (i.e., $O^{\#}$ and $O^{*}$ are independent). Further explanation is given below.

$$
\begin{aligned}
& \theta=\mathrm{P}(O \geq d) \\
& =\mathrm{P}\left(O^{\#} \geq d\right)+\sum_{j=1}^{d} \mathrm{P}\left(O^{\#}=d-j, O^{*} \geq j\right)
\end{aligned}
$$

which, in general, is not equal to $\mathrm{P}\left(O^{\#} \geq d\right)+\sum_{j=1}^{d} \mathrm{P}\left(O^{\#}=d-j\right) \mathrm{P}\left(O^{*} \geq j\right)$, as stated in Equation (14) in [8] and Equation (14) in [9]. The assumption in [8] and [9] states that the regular and rework paths share the same capacity. This leads to that $O^{\#}$ and $O^{*}$ are dependent.

\section{Proposed Analytical Result for One Rework}

In this section, we correct the mistake in Song rule with rework when the rework and non-rework path share the same capacity. That is, this paper proposes correct version of the analytical result for analytical computing $\theta$ for rework case. We claim that notations such $A_{i}$ and $B_{i}$ set up in papers [8] and [9] are helpful and necessary because the correct version for computing $\theta$ can be easily derived using these notations.

$$
\begin{aligned}
& \theta=\mathrm{P}(O \geq d) \\
& =\mathrm{P}\left(O^{\#} \geq d\right)+\sum_{j=1}^{d} \mathrm{P}\left(O^{\#}=d-j, O^{*} \geq j\right)
\end{aligned}
$$

where $\mathrm{P}\left(O^{\#} \geq d\right)$ is derived in Theorem 1 in [8]. The second term $\mathrm{P}\left(O^{\#}=d-j, O^{*} \geq\right.$ $j$ ) can be further decomposed.

Consider the assumption under the rule 3.1 (i.e. the rework and regular path share the same capacity).

- Case 1. $k=1$ (rework items are re-produced starting from workstation 1)

$$
\begin{aligned}
& \mathrm{P} \\
= & \left.\sum^{\#}=d-j, O^{*} \geq j\right) \\
& \sum_{b_{n}=d-j}^{d-j} \sum_{a_{n}=b_{n}}^{b_{0}} \sum_{b_{n-1}=a_{n}}^{b_{0}} \sum_{a_{n-1}=b_{n-1}}^{b_{0}} \ldots \sum_{b_{2}=a_{3}}^{b_{0}} \sum_{a_{2}=b_{2}}^{b_{0}} \sum_{b_{1}=a_{2}}^{b_{0}} \sum_{a_{1}=b_{1}}^{b_{0}} \sum_{b_{n-1}^{*}=j}^{b_{k-1}^{*}} \sum_{a_{n}^{*}=b_{n}^{*}}^{b_{k-1}^{*}} \sum_{b_{n-1}^{*}=a_{n}^{*}}^{b_{k-1}^{*}} \sum_{a_{n-1}^{*}=b_{n-1}^{*}}^{b_{k-1}^{*}} \ldots \sum_{b_{k}^{*}=a_{k+1}^{*}}^{b_{k-1}^{*}} \sum_{a_{k}^{*}=b_{k}^{*}}^{n} I\left(b_{k-1}^{*} \geq j\right) \\
& \prod_{i=k}^{n} \mathrm{P}\left(A_{i}^{*}=a_{i}^{*} \mid B_{i-1}^{*}=b_{i-1}^{*}\right) \mathrm{P}\left(B_{i}^{*}=b_{i}^{*} \mid A_{i}^{*}=a_{i}^{*}\right) \mathrm{P}\left(B_{i}=b_{i} \mid A_{i}=a_{i}\right)
\end{aligned}
$$


- Case 2. $k \geq 2$ (rework items are re-produced starting from workstation $k$ )

$$
\begin{aligned}
& \mathrm{P}\left(O^{\#}=d-j, O^{*} \geq j\right) \\
= & \sum_{b_{n}=d-j}^{d-j} \sum_{a_{n}=b_{n}}^{b_{0}} \sum_{b_{n-1}=a_{n}}^{b_{0}} \sum_{a_{n-1}=b_{n-1}}^{b_{0}} \ldots \sum_{b_{2}=a_{3}}^{b_{0}} \sum_{a_{2}=b_{2}}^{b_{0}} \sum_{b_{1}=a_{2}}^{b_{0}} \sum_{a_{1}=b_{1}}^{b_{0}} \sum_{b_{n}^{*}=j}^{b_{k-1}^{*}} \sum_{a_{n}^{*}=b_{n}^{*}}^{b_{k-1}^{*}} \sum_{b_{n-1}^{*}=a_{n}^{*}}^{b_{k-1}^{*}} \sum_{a_{n-1}^{*}=b_{n-1}^{*}}^{b_{k-1}^{*}} \ldots \sum_{b_{k}^{*}=a_{k+1}^{*}}^{b_{k-1}^{*}} \sum_{a_{k}^{*}=b_{k}^{*}}^{b_{k-1}^{*}} I\left(b_{k-1}^{*} \geq j\right) \\
& {\left[\prod_{i=1}^{k-1} \mathrm{P}\left(A_{i}=a_{i} \mid B_{i-1}=b_{i-1}\right) \mathrm{P}\left(B_{i}=b_{i} \mid A_{i}=a_{i}\right)\right] } \\
& {\left[\prod_{i=k}^{n} \mathrm{P}\left(A_{i}^{*}=a_{i}^{*} \mid B_{i-1}^{*}=b_{i-1}^{*}\right) \mathrm{P}\left(B_{i}^{*}=b_{i}^{*} \mid A_{i}^{*}=a_{i}^{*}\right) \mathrm{P}\left(B_{i}=b_{i} \mid A_{i}=a_{i}\right)\right] }
\end{aligned}
$$

where $b_{k-1}^{*}=a_{r}-b_{r}$

$$
\begin{aligned}
& I\left(b_{k-1}^{*} \geq j\right)= \begin{cases}1, & \text { if } b_{k-1}^{*} \geq j \\
0, & \text { if } b_{k-1}^{*}<j ;\end{cases} \\
& \mathrm{P}\left(A_{i}^{*}=a_{i}^{*} \mid B_{i-1}^{*}=b_{i-1}^{*}\right) \\
& = \begin{cases}\mathrm{P}\left(X_{i}=a_{i}+a_{i}^{*}\right) & \text { if } a_{i}^{*}<b_{i-1}^{*} \\
\mathrm{P}\left(X_{i} \geq a_{i}+a_{i}^{*}\right) & \text { if } a_{i}^{*}=b_{i-1}^{*} \\
0 & \text { if } a_{i}^{*}>b_{i-1}^{*}\end{cases}
\end{aligned}
$$

$i=k, k+1, \ldots, n ;$ and

$$
\begin{aligned}
& \mathrm{P}\left(B_{i}^{*}=b_{i}^{*} \mid A_{i}^{*}=a_{i}^{*}\right)=C_{b_{i}^{*}}^{a_{i}^{*}} \times p_{i}^{b_{i}^{*}}\left(1-p_{i}\right)^{a_{i}^{*}-b_{i}^{*}}, i=k, k+1, \ldots, n, \\
& \mathrm{P}\left(B_{i}=b_{i} \mid A_{i}=a_{i}\right)=C_{b_{i}}^{a_{i}} \times p_{i}^{b_{i}}\left(1-p_{i}\right)^{a_{i}-b_{i}}, i=1,2, \ldots, n,
\end{aligned}
$$

where $C_{b_{i}^{*}}^{a_{i}^{*}}=\frac{a_{i}^{*} !}{b_{i}^{*} !\left(a_{i}^{*}-b_{i}^{*}\right) !}$ and $C_{b_{i}}^{a_{i}}=\frac{a_{i} !}{b_{i} !\left(a_{i}-b_{i}\right) !}$

Due to the space limitation, we do not provide further discussion and examples in this note. More explanation and examples can be seen in [4].

As mentioned in [8] and [9], the Song rule and the proposed corrected version of Song rule in this note are computational inefficiency and are only recommended as a validator instead of a problem solver. For big scale system reliability, simulation approach and the associated leading digit rule (see [6] and [7]) are recommended as the problem solver. 


\section{Summary and Conclusion}

Many systems including manufacturing and production systems can be constructed as stochastic networks with $n$ workstations $\left(\mathrm{WS}_{1}, \mathrm{WS}_{2}, \ldots, \mathrm{WS}_{n}\right)$ in which the stochastic factors include the $\mathrm{WS}_{i}$ 's random capacity and random number of output for each workstation, where $i=1,2, \ldots, n$. Therefore, the system output $O$ is also random variable. The so-called "system reliability" defined as the probability that the output $O$ meets $d$ given input $b, \theta=\mathrm{P}(O \geq d)$, is a critical performance measure for such stochastic systems.

This paper reviews and highlights the drawbacks of the previous works, including twenty one archival publications and the most recent published analytical Song rule. We recommend to only apply the Song rule as a validator instead of a problem solver due to its computational inefficiency. That is, we strongly recommend to use simulation as the problem spotter and problem solver for system reliability, which was aleady proposed in paper [8] and [9].

\section{Acknowledgement}

This material is based upon work supported by the National Science Council under Grant No. MOST 107-2221- E-007-073-MY2. The author would like to thank Prof. D. Engi, Prof. B. Schmeiser, Prof. D. Goldsman, and Prof. Wei-Chang Yeh for helpful discussion.

\section{References}

[1] P.-C. Chang, Reliability of a Maintainable Manufacturing Network subject to Budget, International Journal of Operations Research, 2016, Vol. 13, No. 3, pp. 95-101.

[2] P.-C. Chang, A simulation analysis of the impact of finite buffer storage on manufacturing system reliability, Simulation Modelling Practice and Theory, 2017, Vol. 70, pp. 49-158.

[3] P. C. Chang, Y.K. Line, and J. C. Chen, System reliability for a multi-state manufacturing network with joint buffer stations, Journal of Manufacturing Systems, 2017, Vol. 42, pp. 170-178.

[4] Chia Ti, Huang, A Note Correcting Song Rule for System Reliability with Rework, Master Thesis, National Tsing Hua University, Taiwan, R.O.C., 2019.

[5] Y.-K. Lin, P.-C. Chang, and C. H. Huang, System reliability evaluation of a Multistate Manufacturing Network in H. Pham (ed.) Quality and Reliability Management and its Applications, Springer-Verlag, London, 2016, pp. 117-143.

[6] W.-M. T. Song, and B. Schmeiser, Omitting Meaningless Digits in Point Estimates: the Probability Guarantee of Leading-Digit Rules, Operations Research, 2009, Vol. 57, pp. 109-117.

[7] W.-M. T. Song, and B. Schmeiser, Displaying statistical point estimates using the leading-digit rule, IIE Transaction, 2011, Vol. 43, pp. 851-862.

[8] W.-M. T. Song, Simulation and the Song Rule as Spotters and Validators of Analytical Results ---A Note Correcting "System Reliability Results" in a Review of the Literature, IEEE Transactions on Relaibility, 2017, Vol. 66, No. 4, pp. 1012-1024.

[9] W-M. T. Song, and P. Lin, System Reliability of Stochastic Networks with Multiple Reworks, Reliability Engineering and System Safety, 2018, Vol. 169, pp. 158-168. 